\title{
Resveratrol protects cardiomyocytes from doxorubicin-induced apoptosis through the AMPK/P53 pathway
}

\author{
MI-HUA LIU ${ }^{1 *}$, XIAO-LONG LIN ${ }^{2 *}$, DONG-MING GUO ${ }^{3 *}$, YUAN ZHANG $^{4}$, CONG YUAN $^{5}$, \\ TIAN-PING TAN ${ }^{1}$, YU-DAN CHEN ${ }^{1}$, SHAO-JIAN WU ${ }^{1}$, ZU-FENG YE $^{1}$ and JUN HE ${ }^{1}$ \\ ${ }^{1}$ Department of Clinical Laboratory, Affiliated Nanhua Hospital, University of South China, Hengyang, Hunan 421001; \\ ${ }^{2}$ Department of Pathology, The Third People's Hospital of Huizhou Affiliated to Guangzhou Medical University, Huizhou, \\ Guangdong 516002; ${ }^{3}$ Laboratory of Clinical Anatomy, University of South China, Hengyang, Hunan 421001; \\ ${ }^{4}$ Department of Pathology, Mawangdui Hospital, Changsha, Hunan 410016; ${ }^{5}$ Department of Cardiology, \\ The First Hospital of Changsha, Changsha, Hunan 410005, P.R. China
}

Received January 15, 2015; Accepted November 10, 2015

DOI: $10.3892 / \mathrm{mmr} .2015 .4665$

\begin{abstract}
Doxorubicin (DOX) is an efficient drug used in cancer therapy; however, it has severe cardiotoxic side effects. The aim of the present study was to investigate the effects of resveratrol on the adenosine monophosphate-activated protein kinase (AMPK)/P53 pathway in mediating DOX-induced cytotoxicity. H9c2 cells were exposed to $5 \mu \mathrm{M}$ DOX for $24 \mathrm{~h}$ to establish a model of DOX-induced cardiotoxicity. DOX administration amplified P53 and B-cell lymphoma 2 (Bcl-2)-associated X protein (Bax) expression and decreased $\mathrm{Bcl}-2$ expression in $\mathrm{H} 9 \mathrm{c} 2$ cells. Resveratrol increased the cell viability and decreased the apoptotic rate. In addition, resveratrol markedly increased the phosphorylation of AMPK. Of note, resveratrol protected against DOX-induced increases of P53 and Bax and also prevented the downregulation of Bcl-2 in H9c2 cells. Furthermore, AMPK inhibitor Compound C abolished the protective effects of resveratrol. The results of the present study therefore indicated that resveratrol protected H9c2 cells from DOX-induced apoptosis via the AMPK/P53 pathway.
\end{abstract}

\section{Introduction}

Doxorubicin (DOX) remains one of the most widely used anti-cancer drugs (1); however, its clinical use is limited due

Correspondence to: Mr. Mi-Hua Liu or Mr. Jun He, Department of Clinical Laboratory, Affiliated Nanhua Hospital, University of South China, 336 Dongfeng South Road, Hengyang, Hunan 421001, P.R. China

E-mail: mihualiu@163.com

E-mail: 445186184@qq.com

*Contributed equally

Key words: resveratrol, doxorubicin, apoptosis, P53, adenosine monophosphate-activated protein kinase to its severe cardiotoxicity (2). Spallarossa et al (3) showed that cardiomyocyte apoptosis has an important role in DOX-induced cardiotoxicity. Therefore, exploration of the mechanism of DOX-induced cardiomyocyte apoptosis is required to develop strategies to reduce the side effects of DOX by preventing DOX-induced cardiotoxicity.

Resveratrol is a polyphenol with potent cardioprotective, anti-inflammatory and anti-cancer activities $(4,5)$. Resveratrol is able to decrease the infarct size and reduce apoptosis in ischemia-reperfusion injury (6). Tatlidede et al (7) revealed the protective effect of resveratrol against DOX-induced cardiomyocyte apoptosis. In addition, resveratrol not only improved the anti-cancer activity of DOX, but also exerted cardioprotective effects $(8,9)$. Therefore, combined treatment of resveratrol with DOX may be a feasible strategy to reduce DOX-induced cardiotoxicity (10). However, the underlying mechanisms of how resveratrol exerts its cardioprotective effects against DOX-induced cardiotoxicity have remained to be fully elucidated.

Adenosine monophosphate-activated protein kinase (AMPK) is an key regulatory protein in cellular energy metabolism, and AMPK activation can regulate cell apoptosis (11-13). AMPK activation results in the accumulation of pro-apoptotic protein p53 to induce myocardial-cell apoptosis (14). Chen et al (13) reported that activation of the AMPK/P53 signaling pathway has a crucial role in DOX-induced H9c2 cell death and apoptosis. Furthermore, Liu et al (15) confirmed that inhibition of the AMPK/P53 signal transduction pathway can suppress DOX-stimulated cardiomyocyte apoptosis. In the present study, H9c2 cells were treated with DOX to establish a cell model of chemotherapy-induced cardiotoxicity (16) and explored whether resveratrol inhibits DOX-induced cardiomyocyte apoptosis via the AMPK/P53 pathway.

\section{Materials and methods}

Materials. Hoechst 33258, 3-(4,5-dimethylthiazol-2-yl)-2,5-diphenyltetrazolium bromide (MTT), DOX, resveratrol and AMPK inhibitor compound $\mathrm{C}$ were purchased 
from Sigma-Aldrich (St. Louis, MO, USA). The enhanced chemiluminescence (ECL) solution was purchased from Beyotime Institute of Biotechnology (Haimen, China). All cell culture medium components were purchased from Thermo Fisher Scientific, Inc. (Waltham, MA, USA) unless stated otherwise.

Cell culture. H9c2 cardiac cells were cultured in Dulbecco's modified Eagle's medium (DMEM) supplemented with $10 \%$ fetal bovine serum (FBS) in a humidified atmosphere containing $5 \% \mathrm{CO}_{2}$ at $37^{\circ} \mathrm{C}$. The $\mathrm{H} 9 \mathrm{c} 2$ cardiac myocytes were seeded onto six-well plates at a density of $2 \times 10^{6}$ cells/well and treated as follows to form the different groups: Control group, H9c2 cardiac cells were incubated in $0.5 \%$ FBS DMEM for $24 \mathrm{~h}$; DOX group, treated with DOX $(5 \mu \mathrm{M})$ for $24 \mathrm{~h}$; RES + DOX group, treated with resveratrol (25 $\mu \mathrm{M})$ for 30 min prior to exposure to DOX $(5 \mu \mathrm{M})$ for $24 \mathrm{~h}$; RES + DOX + compound C group, treated with compound C $(10 \mu \mathrm{M})$ for $60 \mathrm{~min}$ prior to stimulation with resveratrol, followed by a 24 -h culture with DOX.

MTT assay. An MTT assay was used to assess cell viability. H9c2 cardiac myocytes $(5,000$ cells/well) were seeded in 96-well microtiter plates. After incubation with AMPK inhibitor compound $\mathrm{C}(10 \mu \mathrm{M})$ for $60 \mathrm{~min}$ and/or resveratrol $(25 \mu \mathrm{M})$ for $30 \mathrm{~min}$, cells were cultured with $5 \mu \mathrm{M}$ DOX for $24 \mathrm{~h}$. Subsequently, $10 \mu \mathrm{l}$ MTT solution $(5 \mathrm{mg} / \mathrm{ml})$ was added to each well, followed by incubation for a further $4 \mathrm{~h}$ at $37^{\circ} \mathrm{C}$. Formazan crystals were dissolved using $150 \mu \mathrm{l}$ dimethyl sulfoxide and the absorbance was measured at $470 \mathrm{~nm}$ (SpectraMax 190 Absorbance Microplate Reader; Molecular Devices LLC, Sunnyvale, CA, USA) and used to calculate the cell viability relative to that of the control group. The assay was performed with each experimental condition run in triplicate.

Assessment of apoptosis by Hoechst 33258 nuclear staining. Hoechst 33258 was used to assess cell apoptosis. Following the above-mentioned treatments to form the various groups, $\mathrm{H} 9 \mathrm{c} 2$ cells were seeded at a density of $2 \times 10^{6}$ cells/well, incubated for $24 \mathrm{~h}$ and fixed in ice-cold $4 \%$ paraformaldehyde (Beyotime Institute of Biotechnology) dissolved in phosphate-buffered saline at $37^{\circ} \mathrm{C}$ for $20 \mathrm{~min}$. Goat serum (5\%; Beyotime Institute of Biotechnology) was used to block non-specific binding. Samples were then incubated with $10 \mu \mathrm{g} / \mathrm{ml}$ Hoechst 33258 at $37^{\circ} \mathrm{C}$ for $15 \mathrm{~min}$. The slides were visualized under a fluorescence microscope (BX50-FLA; Olympus, Tokyo, Japan). Apoptotic cells were identified as those with a nucleus exhibiting brightly stained condensed chromatin or unclear fragments, while viable cells displayed a normal nuclear size and uniform fluorescence.

Western blot analysis. H9c2 cells were directly homogenized with cell lysis buffer (Cell Signaling Technology, Inc., Danvers, MA, USA) with phosphatase inhibitor cocktail (Sigma-Aldrich). Protein extracts were collected following sample centrifugation at $12,000 \mathrm{x}$ g for $10 \mathrm{~min}$ at $4^{\circ} \mathrm{C}$. Equal quantities of extracted proteins were then separated in $10 \%$ sodium dodecyl sulfate-polyacrylamide electrophoresis gels (Beyotime Institute of Biotechnology) and transferred to a polyvinylidene difluoride membrane (Beyotime Institute of Biotechnology). The membranes were incubated in 5\% non-fat dry milk at $37^{\circ} \mathrm{C}$ for $2 \mathrm{~h}$ and the blots were incubated overnight at $4^{\circ} \mathrm{C}$ with the following primary antibodies: Rabbit phosphorylated (p)-AMPK polyclonal antibody (cat. no. 2535; 1:2,000 dilution; Cell Signaling Technology, Inc.), rabbit AMPK monoclonal antibody (cat. no. 5831; 1:1,000 dilution; Cell Signaling Technology, Inc.), rabbit P53 monoclonal antibody (cat. no. ab179477; 1:2,000 dilution; Abcam, Cambridge, MA, USA), rabbit anti-B-cell lymphoma (Bcl-2)-associated $\mathrm{X}$ protein (Bax) polyclonal antibody (cat. no. Ab026; 1:400 dilution; Beyotime Institute of Biotechnology) and rabbit anti-Bcl-2 polyclonal antibody (cat. no. AB112; 1:4,000 dilution; Beyotime Institute of Biotechnology). Following a $30 \mathrm{~min}$ wash, the membranes were subsequently incubated with the appropriate horseradish peroxidase-conjugated secondary antibodies (cat. no. A0208; 1:1,000 dilution; Beyotime Institute of Biotechnology) for $2 \mathrm{~h}$. Protein expression was determined using an enhanced chemiluminescence reagent kit (Beyotime Institute of Biotechnology) and the Tanon-5500 western blotting detection system (Tanon Science and Technology Co., Ltd., Shanghai, China), and quantified using the Quantity One Software Package (version 4.6.2; Bio-Rad Laboratories, Ltd., Hercules, CA, USA).

Statistical analysis. Values are expressed as the mean \pm standard error of the mean. Statistical analysis of data was assessed using one-way analysis of variance with SPSS 13.0 (SPSS, Inc., Chicago, IL, USA). P $<0.05$ was considered to indicate a statistically significant difference.

\section{Results}

DOX decreases the phosphorylation of AMPK and increases the expression of P53 in $\mathrm{H} 9 \mathrm{c} 2$ cells. As shown in Fig. 1A, DOX decreased the phosphorylation of AMPK in H9c2 cells in a concentration-dependent manner at the tested concentrations of 1-10 $\mu \mathrm{M}$. Fig. 1B shows that DOX significantly induced the expression of P53 at $5 \mu \mathrm{M}$, which was further increased at $10 \mu \mathrm{M}$.

Resveratrol increases the phosphorylation of AMPK and decreases the expression of P53 in DOX-induced H9c2 cells. To investigate the effects of resveratrol against DOX-induced toxicity, the phosphorylation of AMPK and the expression of P53 in H9c2 cells were assessed following DOX and resveratrol treatment. Fig. 2 shows that resveratrol increased the phosphorylation of AMPK and decreased the expression of P53 in a concentration-dependent manner in DOX-induced H9c2 cells. The phosphorylation of AMPK was significantly increased by resveratrol at the concentration of $10 \mu \mathrm{M}$ and above.

Compound C inhibits the cardioprotective effects of resveratrol against DOX-mediated decreases in the phosphorylation of AMPK and increases in the expression of P53 in $\mathrm{H} 9 \mathrm{c} 2$ cells. To explore the implication of the AMPK/P53 pathway in the protective effects of resveratrol, $\mathrm{H} 9 \mathrm{c} 2$ cells were pre-treated with AMPK inhibitor compound $\mathrm{C}$ followed by treatment with DOX and resveratrol. The results showed that compound C 
A
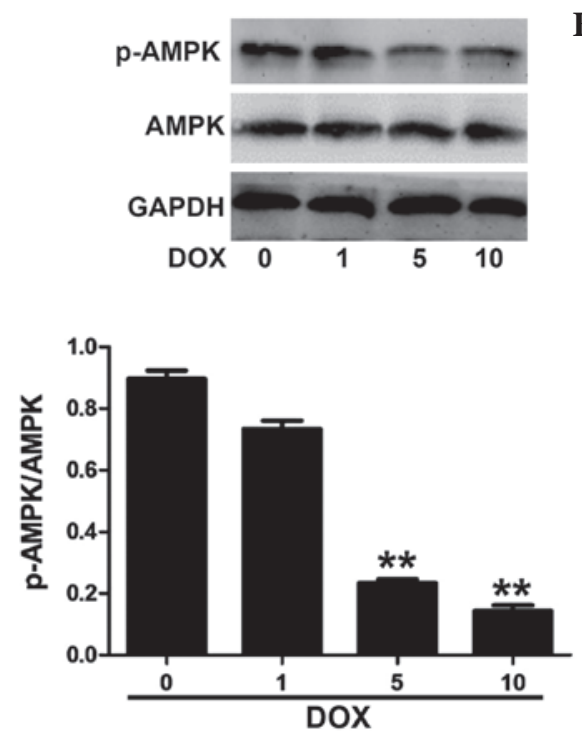

B
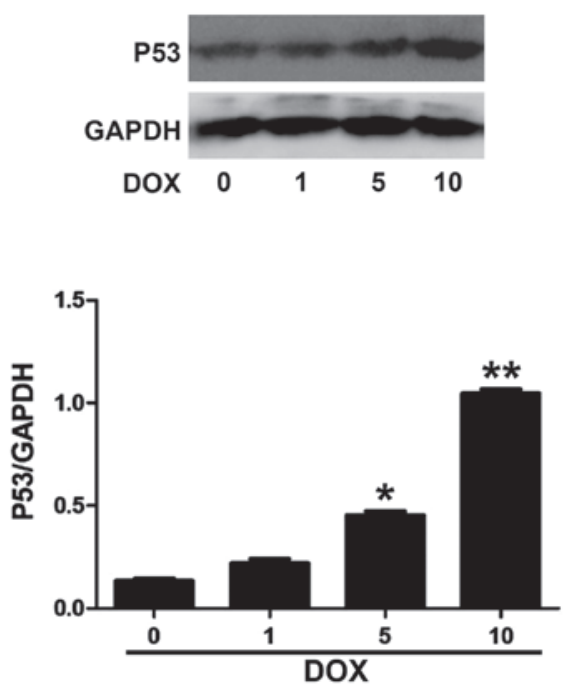

Figure 1. H9c2 cells were treated with $0,1,5,10 \mu \mathrm{M}$ DOX for $24 \mathrm{~h}$. DOX dose-dependently decreases the levels of p-AMPK and P53 in H9c2 cells. (A) AMPK phosphorylation and (B) P53 levels were analyzed by immunoblotting. Relative levels of p-AMPK vs. total AMPK and P53 in each sample as determined by densitometry. Values are expressed as the mean \pm standard error of the mean $(n=3) .{ }^{*} \mathrm{P}<0.05,{ }^{* *} \mathrm{P}<0.01$ vs. the control group. DOX, doxorubicin; $\mathrm{p}-\mathrm{AMPK}$, phosphorylated adenosine monophosphate-activated protein kinase.

A
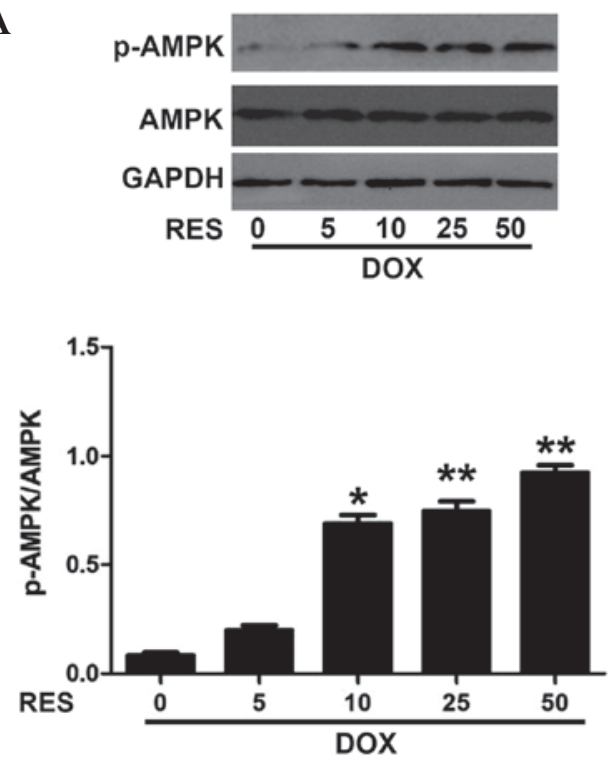

B
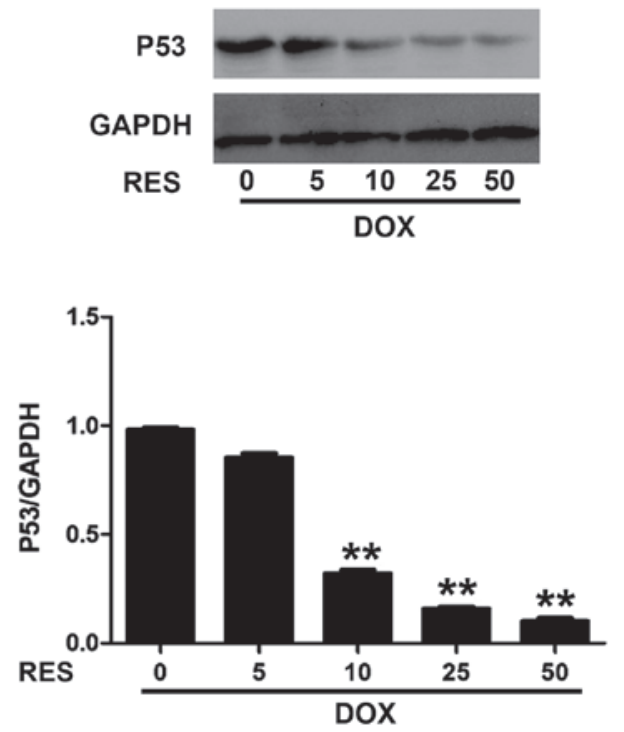

Figure 2. H9c2 cells were treated with $0,5,10,25$ and $50 \mu \mathrm{M}$ RES for 30 min prior to exposure to $5 \mu \mathrm{M}$ DOX for $24 \mathrm{~h}$. RES dose-dependently increases the levels of p-AMPK and P53 in H9c2 cells. (A) AMPK phosphorylation and (B) P53 expression were analyzed by immunoblotting. Relative levels of p-AMPK vs. total AMPK and P53 in each sample as determined by densitometry. Values are expressed as the mean \pm standard error $(\mathrm{n}=3) .{ }^{*} \mathrm{P}<0.05$, ${ }^{* *} \mathrm{P}<0.01$ vs. the control group. DOX, doxorubicin; p-AMPK, phosphorylated adenosine monophosphate-activated protein kinase; RES, resveratrol.

significantly attenuated the inhibitory effects of resveratrol on the DOX-mediated reduction of AMPK phosphorylation and increase of P53 expression (Fig. 3). These results further indicated that the AMPK/P53 pathway was involved in the protective effects of resveratrol.

Resveratrol attenuates DOX-induced reduction of Bcl-2 and enhancement of Bax expression in cardiomyocytes. To further investigate the protective effects of resveratrol against DOX-mediated toxicity in $\mathrm{H} 9 \mathrm{c} 2$ cells, the expression of $\mathrm{Bcl}-2$ and Bax was examined. As illustrated in Fig. 4, DOX markedly decreased the expression of $\mathrm{Bcl}-2$ and increased the expression of Bax. Of note, following pre-treatment with resveratrol, the levels of Bax were decreased, whereas Bcl-2 levels increased, indicating that resveratrol exerted anti-apoptotic effects on DOX-treated H9c2 cells. However, compound C abolished the protective effects of resveratrol.

Resveratrol inhibits DOX-induced cytotoxicity. Fig. 5 shows that DOX treatment significantly decreased the viability of $\mathrm{H} 9 \mathrm{c} 2$ cells and induced marked cytotoxicity. However, pre-treatment with resveratrol significantly increased the cell viability and ameliorated the DOX-induced cytotoxicity. In addition, compound $\mathrm{C}$ abolished the protective effects of 
A
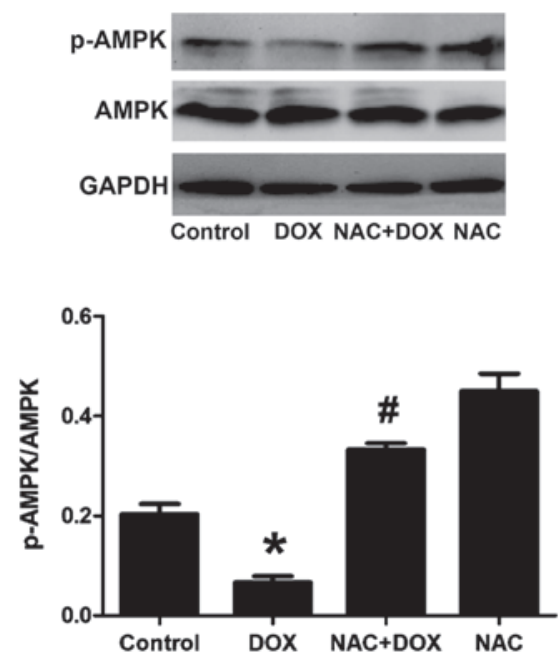

B
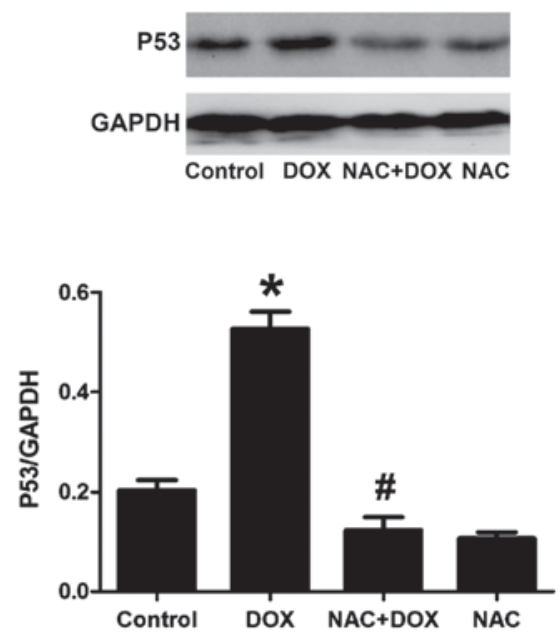

Figure 3. Compound C inhibits the effect of RES on p-AMPK and P53 in H9c2 cells. Control group, H9c2 cardiac cells incubated in $0.5 \%$ FBS DMEM for $24 \mathrm{~h}$; DOX group, treated with DOX $(5 \mu \mathrm{M})$ for $24 \mathrm{~h}$; RES + DOX group, treated with RES $(25 \mu \mathrm{M})$ for 30 min prior to exposure to DOX $(5 \mu \mathrm{M})$ for $24 \mathrm{~h}$; RES + DOX + compound C group, treated with compound C $(10 \mu \mathrm{M})$ for 60 min prior to stimulation with RES, followed by a 24-h culture with DOX. (A) AMPK phosphorylation and (B) P53 expression were analyzed by immunoblotting. Relative levels of p-AMPK vs. total AMPK and P53 in each sample as determined by densitometry. Values are expressed as the mean \pm standard error $(n=3)$. ${ }^{*} \mathrm{P}<0.05$, compared with the control group; ${ }^{*} \mathrm{P}<0.05$, compared with the DOX-treated group. DOX, doxorubicin; RES, resveratrol; p-AMPK, phosphorylated adenosine monophosphate-activated protein kinase.

A
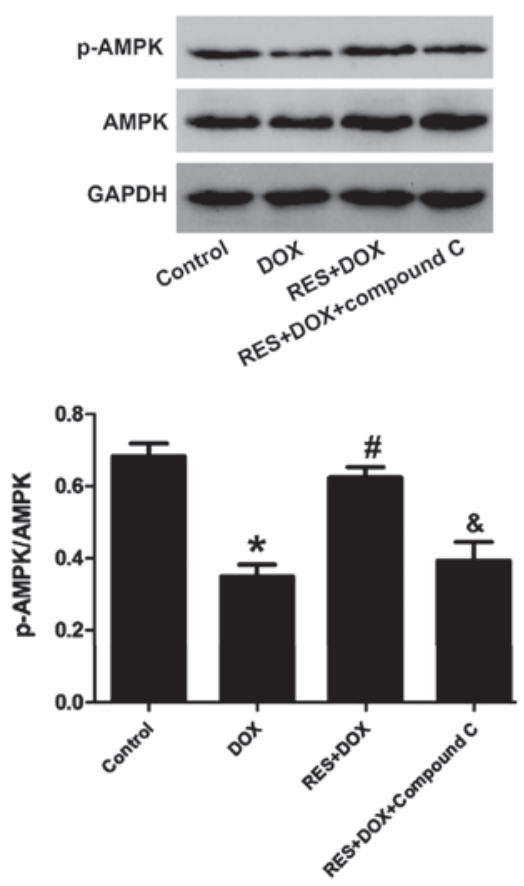

B
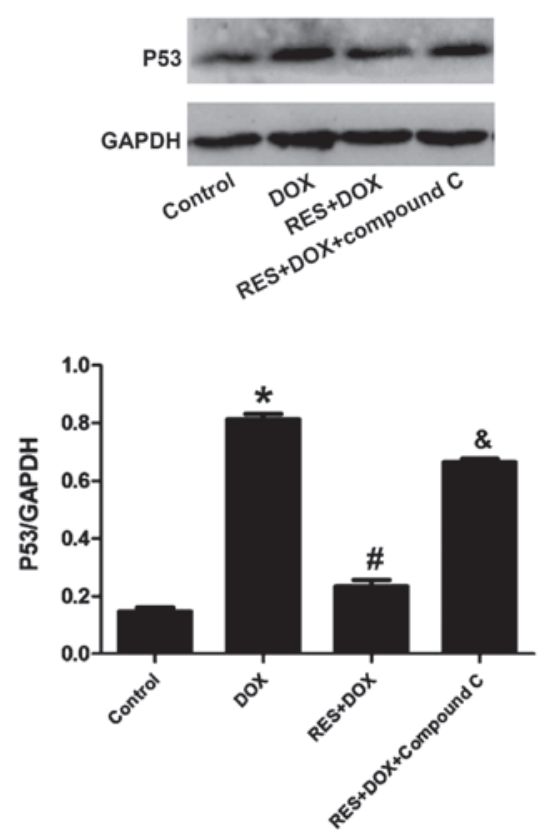

Figure 4. Effects of RES on the changes of Bcl-2 and Bax expression induced by DOX in H9c2 cells. Control group, H9c2 cardiac cells incubated in 0.5\% FBS DMEM for $24 \mathrm{~h}$; DOX group, treated with DOX $(5 \mu \mathrm{M})$ for $24 \mathrm{~h}$; RES + DOX group, treated with RES $(25 \mu \mathrm{M})$ for 30 min prior to exposure to DOX $(5 \mu \mathrm{M})$ for $24 \mathrm{~h}$; RES + DOX + compound C group, treated with compound C $(10 \mu \mathrm{M})$ for 60 min prior to stimulation with RES, followed by a 24-h culture with DOX. (A) Western blots demonstrate the expression changes of Bcl-2 and Bax protein. (B) Quantification of data from A by densitometric analysis. Values are expressed as the mean \pm standard error $(n=3)$. ${ }^{*} \mathrm{P}<0.05$, compared with the control group; ${ }^{*} \mathrm{P}<0.05$, compared with the DOX-treated group; ${ }^{\text {P }} \mathrm{P}<0.05$, compared with the RES + DOX group. DOX, doxorubicin; RES, resveratrol; Bcl-2, B-cell lymphoma 2; Bax, Bcl-2-associated X protein.

resveratrol as indicated by decreased cell viability and the induction of marked cytotoxicity.

Resveratrol reduces DOX-induced apoptosis in $\mathrm{H} 9 \mathrm{c} 2$ cells. Fig. 6 shows that DOX induced apoptosis in a large percentage of $\mathrm{H} 9 \mathrm{c} 2$ cells. However, resveratrol inhibited DOX-induced H9c2-cell apoptosis. Pre-treatment of the H9c2 cells with compound $\mathrm{C}$ abolished the protective effects of resveratrol.

\section{Discussion}

Although studies on DOX-derived cardiotoxicity have been performed for decades (17), the underlying mechanisms have remained to be fully elucidated. It is known that oxidative stress-induced cardiomyocyte apoptosis and death is an major molecular mechanism involved in DOX-induced cardiotoxicity $(18,19)$. The present study observed that cell viability 


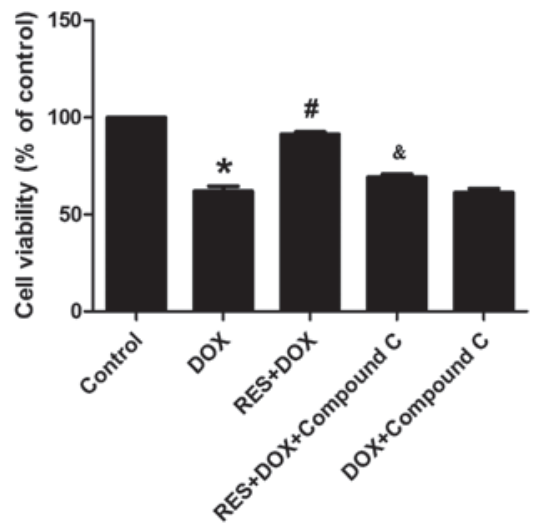

Figure 5. Resveratrol protects H9c2 cells against DOX-induced cytotoxicity. Cell viability was measured using an MTT assay. Values are expressed as the mean \pm standard error of the mean $(n=3)$. ${ }^{*} \mathrm{P}<0.05$, compared with the control group; ${ }^{\text {P }}<0.05$, compared with the DOX-treated group; ${ }^{\circledR} \mathrm{P}<0.05$, compared with the RES + DOX group. DOX, doxorubicin; RES, resveratrol.
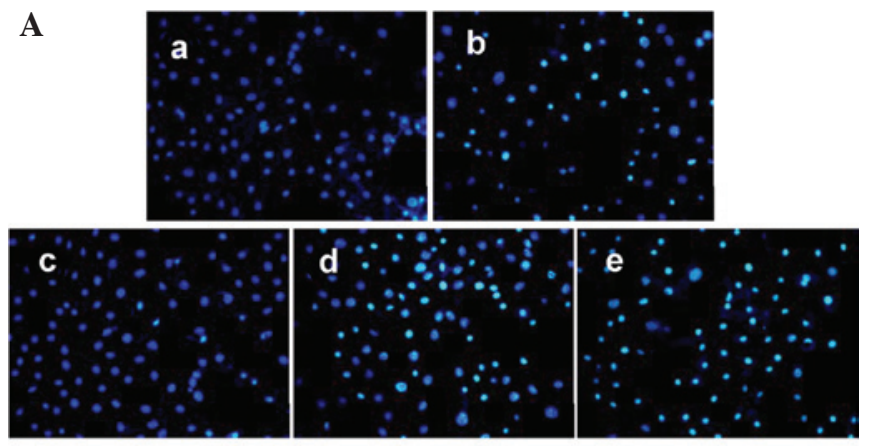

B

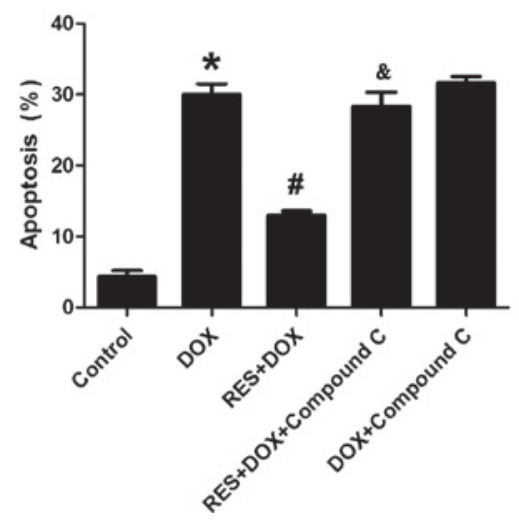

Figure 6. RES reduces DOX-induced apoptosis in $\mathrm{H} 9 \mathrm{c} 2$ cells. (A) Hoechst 33258 nuclear staining followed by fluorescence imaging were performed to observe cell apoptosis. (a) Control group; (b) DOX group; (c) RES + DOX group; (d) RES + DOX + Compound C group; (e) DOX + Compound C group. Magnification, $x 40$. (B) The apoptotic rate was analyzed using a cell counter. Values are expressed as the mean \pm standard error $(\mathrm{n}=5)$. ${ }^{*} \mathrm{P}<0.05$, compared with the control group; ${ }^{\text {"P}} \mathrm{P}<0.05$, compared with the DOX-treated group; ${ }^{\circledR} \mathrm{P}<0.05$, compared with the RES + DOX group. DOX, doxorubicin; RES, resveratrol.

was markedly decreased and cell apoptosis was significantly increased following DOX-induced cellular injury.

Resveratrol potently protects cardiomyocytes from apoptosis and reduces the risk of cardiovascular disease (20). Oktem et al (21) reported that resveratrol protects cardiomyocytes from DOX-induced apoptosis. In addition, resveratrol was shown to enhance the anti-cancer effects of
DOX and to simultaneously protect against DOX-induced cardiotoxicity (9). However, the underlying mechanism of the cardioprotective effects of resveratrol has remained to be fully elucidated.

Shirwany et al (22) have reported that AMPK inhibits the proliferation of non-malignant cells. P53 is a member of the P53 tumor suppressor family and acts as a critical regulator of numerous cellular processes, including cell-cycle arrest and apoptosis (23). Studies have reported that the AMPK/P53 pathway has an important role in DOX-induced cardiomyocyte death $(24,25)$. The present study found that the phosphorylation of AMPK protein was significantly decreased, while the expression of P53 protein was significantly increased in DOX-treated $\mathrm{H} 9 \mathrm{c} 2$ cells. In addition, the expression of Bax protein was significantly increased, while the expression of $\mathrm{Bcl}-2$ protein was markedly reduced in DOX-treated H9c2 cells. These results supported that the AMPK/P53 pathway was involved in DOX-induced cardiomyocyte apoptosis.

To further elucidate the molecular mechanism of the cardioprotective effects of resveratrol, its effect on the AMPK/P53 pathway was assessed in DOX-treated H9c2 cells. The results demonstrated that resveratrol significantly attenuated DOX-induced decreases in AMPK activation and increases of P53 expression. In addition, resveratrol significantly reduced the DOX-induced enhancement of Bax and the decrease of Bcl-2 protein levels in H9c2 cells. Furthermore, the present study indicated an association of ROS and the deactivation of AMPK in DOX-treated H9c2 cells. Oxidative stress is defined as an imbalanced redox state in which pro-oxidants overwhelm antioxidant capacity, resulting in increased ROS production. ROS has been implicated in the pathogenesis of DOX-induced H9c2 cardiomyocyte apoptosis. AMPK, which is a cellular energy sensor and regulator, as well as a pressure sensor, regulates ROS/redox balance, cell apoptosis and cell proliferation to maintain cellular homeostasis. Thus, it was hypothesized in the present study that resveratrol protects against DOX-induced H9c2 cardiomyocyte apoptosis via reduce generation of ROS, which activates AMPK and the expression of p53 protein. In addition, compound $\mathrm{C}$, an inhibitor of AMPK phosphorylation, reversed the protective effects of resveratrol by significantly increasing apoptosis of $\mathrm{H} 9 \mathrm{c} 2$ cells, inhibiting the phosphorylation of AMPK and increasing the expression of P53. These results indicated that resveratrol inhibited the generation of ROS and thereby activated AMPK to prevent DOX-induced cardiotoxicity.

In conclusion, the present study showed that resveratrol suppressed DOX-induced cardiomyocyte apoptosis via increasing AMPK phosphorylation and inhibiting P53 expression, as well as inducing Bcl-2 and downregulating Bax expression. These results suggested that resveratrol represents a promising, novel drug candidate for the treatment and prevention of DOX-induced cardiotoxicity.

\section{Acknowledgements}

This work was supported by a grant from the Graduate Student Research Innovation Project of Hunan province (grant no. CX2013B397). 


\section{References}

1. Menna P, Recalcati S, Cairo G and Minotti G: An introduction to the metabolic determinants of anthracycline cardiotoxicity. Cardiovasc Toxicol 7: 80-85, 2007.

2. Lipshultz SE, Karnik R, Sambatakos P, Franco VI, Ross SW and Miller TL: Anthracycline-related cardiotoxicity in childhood cancer survivors. Curr Opin Cardiol 29: 103-112, 2014.

3. Spallarossa P, Garibaldi S, Altieri P, Fabbi P, Manca V, Nasti S, Rossettin P, Ghigliotti G, Ballestrero A, Patrone F, et al: Carvedilol prevents doxorubicin-induced free radical release and apoptosis in cardiomyocytes in vitro. J Mol Cell Cardiol 37: 837-846, 2004.

4. Renaud J, Bournival J, Zottig X and Martinoli MG: Resveratrol protects DAergic PC12 cells from high glucose-induced oxidative stress and apoptosis: Effect on p53 and GRP75 localization. Neurotox Res 25: 110-123, 2014.

5. Liu MH, Yuan C, He J, Tan TP, Wu SJ, Fu HY, Liu J, Yu S, Chen YD, Le QF, et al: Resveratrol protects PC12 cells from high glucose-induced neurotoxicity Via PI3K/Akt/FoxO3a pathway. Cell Mol Neurobiol 35: 513-522, 2015.

6. Das S, Falchi M, Bertelli A, Maulik N and Das DK: Attenuation of ischemia/reperfusion injury in rats by the anti-inflammatory action of resveratrol. Arzneimittelforschung 56: 700-706, 2006.

7. Tatlidede E, Sehirli O, Velioğlu-Oğünc A, Cetinel S, Yeğen BC, Yarat A, Süleymanoğlu S and Sener G: Resveratrol treatment protects against doxorubicin-induced cardiotoxicity by alleviating oxidative damage. Free Radic Res 43: 195-205, 2009.

8. Shankar S, Singh G and Srivastava RK: Chemoprevention by resveratrol: Molecular mechanisms and therapeutic potential. Front Biosci 12: 4839-4854, 2007.

9. Rezk YA, Balulad SS, Keller RS and Bennett JA: Use of resveratrol to improve the effectiveness of cisplatin and doxorubicin: Study in human gynecologic cancer cell lines and in rodent heart. Am J Obstet Gynecol 194: e23-e26, 2006.

10. Park DG: Antichemosensitizing effect of resveratrol in cotreatment with oxaliplatin in HCT116 colon cancer cell. Ann Surg Treat Res 86: 68-75, 2014

11. Chen L, Xu B, Liu L, Luo Y, Yin J, Zhou H, Chen W, Shen T, Han X and Huang S: Hydrogen peroxide inhibits $\mathrm{mTOR}$ signaling by activation of AMPKalpha leading to apoptosis of neuronal cells. Lab Invest 90: 762-773, 2010.

12. Towler MC and Hardie DG: AMP-activated protein kinase in metabolic control and insulin signaling. Circ Res 100: 328-341, 2007.

13. Chen MB, Wu XY, Gu JH, Guo QT, Shen WX and Lu PH: Activation of AMP-activated protein kinase contributes to doxorubicin-induced cell death and apoptosis in cultured myocardial H9c2 cells. Cell Biochem Biophys 60: 311-322, 2011
14. Okoshi R, Ozaki T, Yamamoto H, Ando K, Koida N, Ono S, Koda T, Kamijo T, Nakagawara A and Kizaki H: Activation of AMP-activated protein kinase induces p53-dependent apoptotic cell death in response to energetic stress. J Biol Chem 283: 3979-3987, 2008.

15. Liu J, Mao W, Ding B and Liang CS: ERKs/p53 signal transduction pathway is involved in doxorubicin-induced apoptosis in H9c2 cells and cardiomyocytes. Am J Physiol Heart Circ Physiol 295: H1956-H1965, 2008.

16. Guo R, Lin J, Xu W, Shen N, Mo L, Zhang C and Feng J: Hydrogen sulfide attenuates doxorubicin-induced cardiotoxicity by inhibition of the p38 MAPK pathway in H9c2 cells. Int J Mol Med 31: 644-650, 2013.

17. Gu J, Hu W and Zhang DD. Resveratrol, a polyphenol phytoalexin, protects against doxorubicin-induced cardiotoxicity. J Cell Mol Med 19: 2324-2328.

18. Wang X, Wang XL, Chen HL, Wu D, Chen JX, Wang XX, Li RL, He JH, Mo L, Cen X, et al: Ghrelin inhibits doxorubicin cardiotoxicity by inhibiting excessive autophagy through AMPK and p38-MAPK. Biochem Pharmacol 88: 334-350, 2014.

19. Guo R, Wu K, Chen J, Mo L, Hua X, Zheng D, Chen P, Chen G, $\mathrm{Xu} \mathrm{W}$ and Feng J: Exogenous hydrogen sulfide protects against doxorubicin-induced inflammation and cytotoxicity by inhibiting p38MAPK/NFkB pathway in H9c2 cardiac cells. Cell Physiol Biochem 32: 1668-1680, 2013.

20. Das DK, Mukherjee S and Ray D: Erratum to: Resveratrol and red wine, healthy heart and longevity. Heart Fail Rev 16: 425-435, 2011.

21. Oktem G, Uysal A, Oral O, Sezer ED, Olukman M, Erol A, Akgur SA and Bilir A: Resveratrol attenuates doxorubicin-induced cellular damage by modulating nitric oxide and apoptosis. Exp Toxicol Pathol 64: 471-479, 2012.

22. Shirwany NA and Zou MH: AMPK: A cellular metabolic and redox sensor. A minireview. Front Biosci (Landmark Ed) 19: 447-474, 2014.

23. Vogelstein B, Lane D and Levine AJ: Surfing the p53 network. Nature 408: 307-310, 2000

24. Wang S, Song P and Zou MH: Inhibition of AMP-activated protein kinase alpha (AMPK $\alpha$ ) by doxorubicin accentuates genotoxic stress and cell death in mouse embryonic fibroblasts and cardiomyocytes: Role of p53 and SIRT1. J Biol Chem 287: 8001-8012, 2012.

25. Jones RG, Plas DR, Kubek S, Buzzai M, Mu J, Xu Y, Birnbaum MJ and Thompson CB: AMP-activated protein kinase induces a p53-dependent metabolic checkpoint. Mol Cell 18: 283-293, 2005. 